\title{
Canine Vertebral Screw and Rod Fixation System: Design and Mechanical Testing
}

\author{
Pakthorn Lewchalermwong ${ }^{1}$ Nirut Suwanna ${ }^{1}$ \\ ${ }^{1}$ Neurology Center, Veterinary Teaching Hospital, Kasetsart \\ University, Bangkok, Thailand \\ 2 Department of Clinical Sciences of Companion Animals, \\ Utrecht University, Utrecht, The Netherlands
}

\author{
Björn P. Meij ${ }^{2}$
}

Vet Comp Orthop Traumatol 2018;31:95-101.

\begin{abstract}
Address for correspondence Pakthorn Lewchalermwong, DVM, MS Diplomate TBVS, Neurology Center, Veterinary Teaching Hospital, Kasetsart University, 50 Ngamwongwan, Bangkok 10900, Thailand (e-mail: I_pakthorn@yahoo.com).
\end{abstract}

\begin{abstract}
Objectives To develop the canine vertebral screw and rod fixation system (CVSRF) and to compare the biomechanical properties between CVSRF and the screw and polymethylmethacrylate (Screw-PMMA) technique for internal fixation of the vertebral column in dogs.

Methods The CVSRF consisted of vertebral screws with monoaxial side-loaded head, rods and specific inner screws connecting rod to the screw head. The CVSRF prototype was made from titanium alloy and manufactured by the rapid prototype machine. Vertebrectomy models were simulated by ultra-high-molecular-weight polyethylene blocks and tested with the CVSRF system $(n=8)$ and the Screw-PMMA technique $(n=8)$. The models were developed according to the American Society for Testing and Materials (ASTM F-1717-04). The biomechanical parameters were the compressive bending yield load, the compressive bending stiffness, the compressive ultimate load and the load displacement curve.

Keywords

Results The mean values of the compressive bending yield load, compressive bending stiffness and compressive ultimate load of the CVSRF were significantly higher than those of the Screw-PMMA technique $(p<0.01)$. The load displacement curve of the CVSRF showed higher rigidity and durability than that of the Screw-PMMA technique.

- canine

- spine

- pedicle screw and rod fixation

- vertebral screw used for canine vertebral stabilization and the biomechanical properties were better than those for the Screw-PMMA device.
\end{abstract}

\section{Introduction}

Spinal implants that are designed for humans and are used for vertebral column fixation in dogs have several limitations. Differences in dog breeds and sizes are reflected in differences in vertebral column anatomy and most human spinal implants are too large for the canine spine. There is only a very limited range of specific spine implants in the market for dogs, and the high costs of spinal implants that are designed for humans prevent their routine use in the dog. Various other surgical techniques have been employed for vertebral column fixation in dogs including modified segmental lumbar plates and screw or pins in combination with polymethylmethacrylate (PMMA) bridges. Pin in conjunction with PMMA and screws combined with PMMA (Screw-PMMA) have been widely used as the gold standard for spinal fixation in dogs due to the lack of commercially available alternatives and their low material costs. However, these techniques have shown treatment failures in canine patients because the size of some devices was inappropriate, realignment of the spine after application was difficult or impossible, and advanced imaging with magnetic resonance 
imaging with the implants in place was hampered with imaging artefacts. Also, PMMA techniques came with postoperative complications like difficulty with wound closure or compression of adjacent soft-tissue structures due to its spaceoccupying effect. ${ }^{1}$

The gold standard for spinal fixation in humans has been pedicle screw and rod fixation system, which has been widely used for more than 30 years. Especially in the lumbar region, surgical approaches like anterior, posterior or transforaminal lumbar intervertebral fusion using pedicle and intervertebral cages have become the standard of care for spinal fusion in humans. ${ }^{2}$ The pedicle screw and rod fixation system ${ }^{3}$ and the pedicle screw and rod fixation system with a titanium cage ${ }^{4}$ were successfully tested ex vivo and applied in vivo ${ }^{5,6}$ in the lumbosacral area of large breed dogs using the smallest pedicle screws available for humans demonstrating that the principle of pedicle screw and rod fixation system may also be used in dogs.

To make the screw-rod system more widely available for dogs of various sizes, this study presents a new implant specific to the canine spine and the results of mechanical testing in a laboratory environment according to a standard testing protocol for human spine implants. The first aim of this study was to design a modern canine vertebral screw and rod fixation (CVSRF) system for internal fixation of the vertebral column in dogs and then build the prototype. The second aim was to mechanically test the prototype construct and compare it with the Screw-PMMA construct.

\section{Materials and Methods}

\section{Design of the Prototype Construct}

Eight samples of the prototype construct of the CVSRF system were manufactured from titanium alloy (Ti 6Al-4V
ELI) and one complete sample included four titanium vertebral screws, two titanium rods, and four titanium inner screws that locked the rods into the screw heads (-Fig. 1). All components of the prototype device were manufactured with a computer numerical control machine (TORNOS/Swiss ST-26) with the highest grade of accuracy for manufacturing medical implants for humans.

The titanium vertebral screw was developed as a selftapping cancellous screw type with monoaxial side-loaded head for receipt of the rod. The outer shape of the screw shaft was cylindrical with a diameter of $3.5 \mathrm{~mm}$ and the inner core was conical with diameter of $2.5 \mathrm{~mm}$ (-Fig. 1A). All screws had a length of $16 \mathrm{~mm}$ resembling the corridor in the thoracolumbar and lumbar vertebral column in dogs. The thread type on the shaft was buttress thread with a pitch of $2.0 \mathrm{~mm}$. The screws were inserted with a custom-made screw inserter that was fitted into the vertebral screw head.

The titanium inner screw had a universal hexagonal M5 screw head design, with a thread pitch of $0.8 \mathrm{~mm}$, a length of $4.0 \mathrm{~mm}$, and the tip had a cone shape with an angle of 120 degrees (-Fig. 1B). The inner screw was inserted in the monoaxial side-loaded head of the vertebral screw with a hexagonal screwdriver of $2.5 \mathrm{~mm}$.

The titanium rods had a standardized diameter of $4.0 \mathrm{~mm}$ and a length of $45 \mathrm{~mm}$ (-Fig. 1C).

\section{Mechanical Testing of the Prototype}

Two ultra-high-molecular-weight polyethylene (UHMWPE) blocks measuring $75 \mathrm{~mm} \times 45 \mathrm{~mm} \times 19 \mathrm{~mm}$ were manufactured to mimic two segments (vertebrae) of the canine vertebral column ( - Fig. 2). Sixteen UHMWPE blocks were needed for testing eight samples of the prototype device. Two vertebral screws per UHMWPE block were manually inserted $40 \mathrm{~mm}$

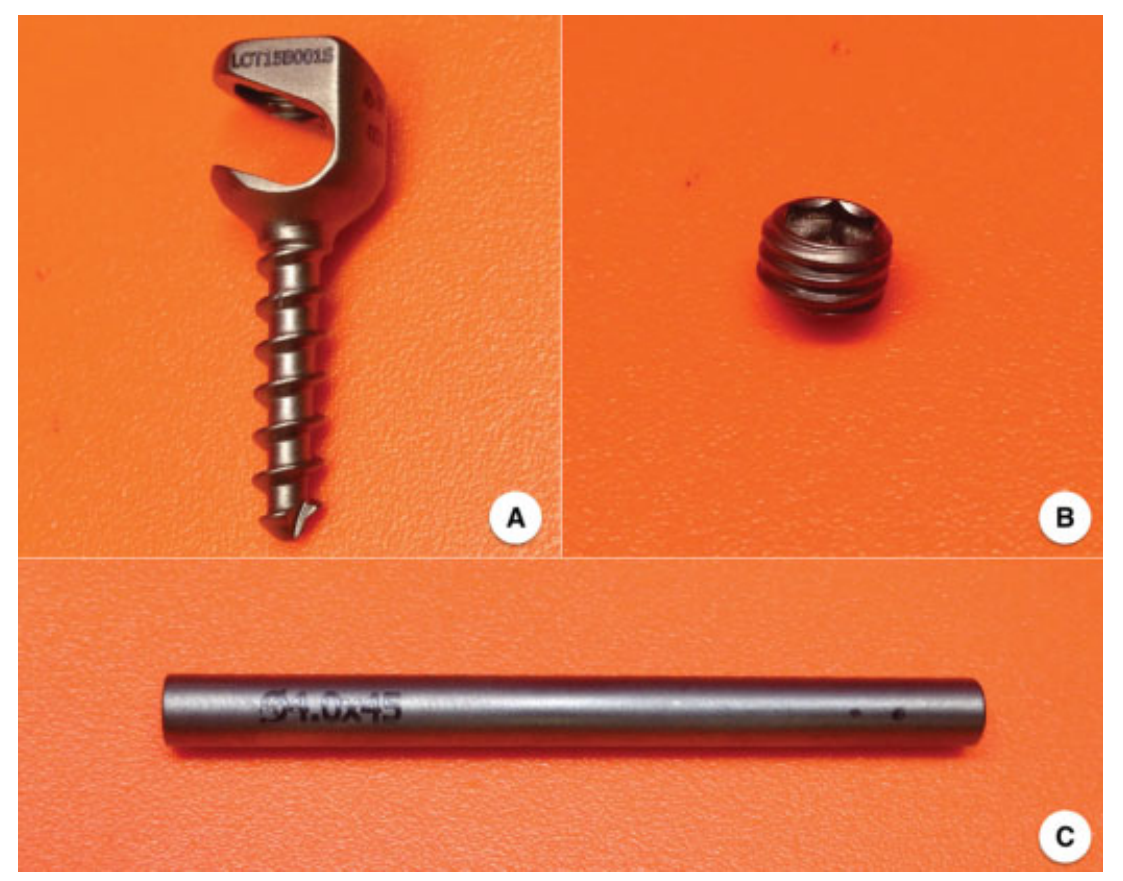

Fig. 1 The canine vertebral screw and rod fixation prototype is manufactured from titanium alloy (Ti-6AI-4V ELI) and each sample includes (A) four vertebral screws (diameter: $3.5 \mathrm{~mm}$, length: $16.0 \mathrm{~mm}$ ), (B) four inner screws (universal hexagonal M5 head, pitch: $0.8 \mathrm{~mm}$, length: $4 \mathrm{~mm}$ ), and (C) two rods (diameter: $4.0 \mathrm{~mm}$, length: $45.0 \mathrm{~mm}$ ). 


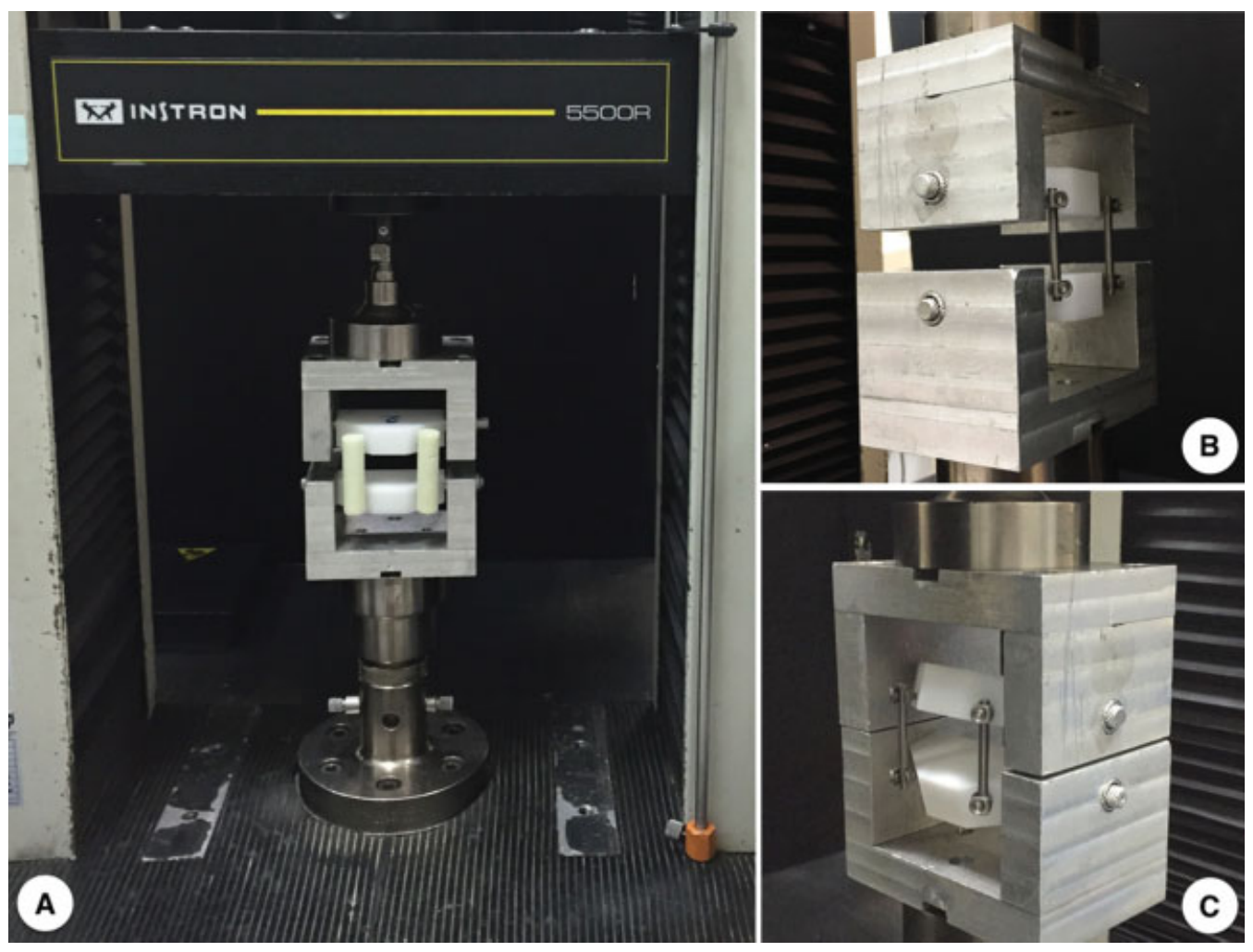

Fig. 2 (A) Setup of the single column-type universal Instron testing machine loaded with the spinal implant assembly. (B) The canine vertebral screw and rod fixation construct including the ultra-high-molecular-weight polyethylene blocks were mounted in U-shaped metal moment arms, $15 \mathrm{~mm}$ apart, and were compressed at a load rate of $5 \mathrm{~mm} / \mathrm{min}$. (C) The compressive ultimate load of canine vertebral screw and rod fixation mechanical testing was reached at $14 \mathrm{~mm}$ displacement when the metal moment arms were $1 \mathrm{~mm}$ apart.

apart using the custom-made screw inserter. The screw shaft was inserted at a depth of $16 \mathrm{~mm}$ leaving the screw head exposed. Two rods were mounted sideways on the vertebral screw heads, perpendicular to the screw shaft and perpendicular to the UHMWPE blocks, connecting the blocks on each side (-Fig. 2). The inner screw was inserted with a 2.5-hexagonal screwdriver and locked the rods in place and completed the spinal implant assembly. The distance between the UHMWPE blocks was $23 \mathrm{~mm}$. The UHMWPE blocks with spinal implant assembly fitted inside the $\mathbf{U}$-shaped metal moment arm and were connected to a roller (-Fig. 2B, C). The U-shaped metal moment arms were $15 \mathrm{~mm}$ apart. The U-shaped metal device with spinal implant assembly was mounted in a single column-type universal testing machine (Instron 55R4502; MTEC, Bangkok, Thailand; -Fig. 2A).

The standard test protocol published by the American Society for Testing and Materials (ASTM F1717-04 International, West Conshohocken, Pennsylvania, United States) for testing spinal implant constructs in a vertebrectomy model was used to measure construct rigidity. Static compressive bending force was applied at a point $35 \mathrm{~mm}$ from each screw insertion point perpendicular to the rod ( - Fig. 2B, C). The bending force was constantly applied at the longitudinal midline of the spinal implant assembly resembling two vertebral bodies connected by an intervertebral disc. The test apparatus was loaded at a rate of $5 \mathrm{~mm} / \mathrm{min}$ at room temperature $\left(24^{\circ} \mathrm{C}\right)$. The static compressive bending force was terminated at $14 \mathrm{~mm}$ displacement of the $\mathbf{U}$-shaped metal moment arms just before they made contact with each other and were $1 \mathrm{~mm}$ apart.

Read-out parameters were visualized as the load-displacement curve that included the compressive bending yield load which was recorded at set intervals, and the compressive bending ultimate load. The compressive bending yield load was defined as the force that caused a displacement at $2 \%$ offset yield and produced permanent deformation (ASTM F1717-04). The compressive bending ultimate load was defined as the maximum force that caused failure of the construct. The compressive bending stiffness was calculated from the load-displacement curve using the compressive bending yield load divided by elastic displacement.

The CVSRF prototype construct was compared with the Screw-PMMA technique that is considered as the gold standard technique in canine internal spinal fixation (-Fig. 2A). For the Screw-PMMA technique, a cancellous screw was chosen with diameter of $4.0 \mathrm{~mm}$ and length of $26 \mathrm{~mm}$. All screws were manufactured from stainless steel 316L (MDC, Bangkok, Thailand) and inserted in each side of the UHMWPE blocks until a depth of 16 and $40 \mathrm{~mm}$ apart. The screw head and $10-\mathrm{mm}$ screw shaft were left exposed to be embedded in PMMA. Two screws on different UHMWPE blocks were embedded in a standardized PMMA (Biomet Bone Cement) bridge that had a diameter of $15 \mathrm{~mm}$ and a length of $55 \mathrm{~mm}$. Also in this set up, the UHMWPE blocks were $23 \mathrm{~mm}$ apart ( - Fig. 2A). The PMMA was prepared as per manufacturer's instructions by mixing $40 \mathrm{~g}$ polymer powder with $20 \mathrm{~mL}$ 
Table 1 Mean $( \pm S D)$ values of static compressive bending forces between the CVSRF $(n=8)$ system and the ScrewPMMA $(n=8)$ technique tested in a vertebrectomy model

\begin{tabular}{|l|l|l|l|}
\hline \multirow{2}{*}{ Groups } & \multicolumn{3}{|l|}{ Mechanical parameters } \\
\cline { 2 - 4 } & $\begin{array}{l}\text { Bending } \\
\text { yield } \\
\text { load }(N)\end{array}$ & $\begin{array}{l}\text { Bending } \\
\text { stiffness } \\
(N / m m)\end{array}$ & $\begin{array}{l}\text { Bending } \\
\text { ultimate } \\
\text { load }(N)\end{array}$ \\
\hline CVSRF & $85.79 \pm 8.23^{\mathrm{a}}$ & $20.75 \pm 4.06^{\mathrm{a}}$ & $166.40 \pm 25.19^{\mathrm{a}}$ \\
\hline $\begin{array}{l}\text { Screw- } \\
\text { PMMA }\end{array}$ & $61.51 \pm 3.73$ & $14.49 \pm 0.53$ & $83.23 \pm 1.84$ \\
\hline
\end{tabular}

Abbreviations: CVSRF, canine vertebral screw and rod fixation; PMMA, polymethylmethacrylate.

${ }^{\mathrm{a}} \mathrm{p}<0.01$ between CVSRF and Screw-PMMA.

methyl methacrylate liquid for each side, and after shaping the bridge, the PMMA was left to polymerize for 10 minutes. Eight samples of the Screw-PMMA construct were measured in the same manner as described earlier for the CVSRF prototype construct. All implants were new before testing and had not been used previously.

\section{Radiography}

Following the mechanical tests of the CVSRF prototype and the Screw-PMMA construct, each spinal implant assembly underwent radiography to assess mode of failure of each construct after testing.

\section{Statistical Analysis}

Statistical analysis was performed using SPSS 17.0 (SPSS, Inc., Chicago, Illinois, United States). The compressive bending yield load, bending stiffness and compressive bending ultimate load data were expressed as mean \pm standard deviation (SD). The CVSRF prototype construct $(n=8)$ was compared with the Screw-PMMA $(n=8)$ and analysed by the independent Student's $t$-test. A $p$-value of $<0.05$ was considered statistically significant.

\section{Results}

\section{Design of the Prototype Construct}

The CVSRF prototype was lightweight and easy to assemble and the screws were inserted with the custom-made screw inserter. The inner screws locked the rods in place and no loosening of rods from the screw heads was observed during and after testing.

\section{Mechanical Testing of the Prototype}

The mechanics of the spinal implant constructs was evaluated with the static compressive bending force according to the ASTM F1717-04 protocol. The mean $( \pm \mathrm{SD})$ values of the compressive bending yield load, the compressive bending stiffness, and compressive ultimate load of CVSRF $(85.79 \pm 8.23 \mathrm{~N}, \quad 20.75 \pm 4.06 \mathrm{~N}$ and $166.40 \pm 25.19 \mathrm{~N}$, respectively) were significantly higher $(p<0.01)$ than those of the Screw-PMMA technique $(61.51 \pm 3.73 \mathrm{~N}, \quad 14.49 \pm 0.53 \mathrm{~N}$ and $83.23 \pm 1.84 \mathrm{~N}$, respectively; - Table 1 ). The load displacement curve of the CVSRF system showed higher rigidity and toughness than that of the Screw-PMMA technique (-Fig. 3 ).

\section{Radiography}

The constructs of the CVSRF system and the Screw-PMMA technique all showed permanent deformation when the compressive bending ultimate load was exceeded ( - Fig. 4). Radiographs after mechanical testing revealed that the permanent deformation occurred due to bending of the screws at their insertion point (-Fig. 5). However, the deformation of the screws in the Screw-PMMA constructs showed more bending than those in the CVSRF prototype (-Fig. 5).

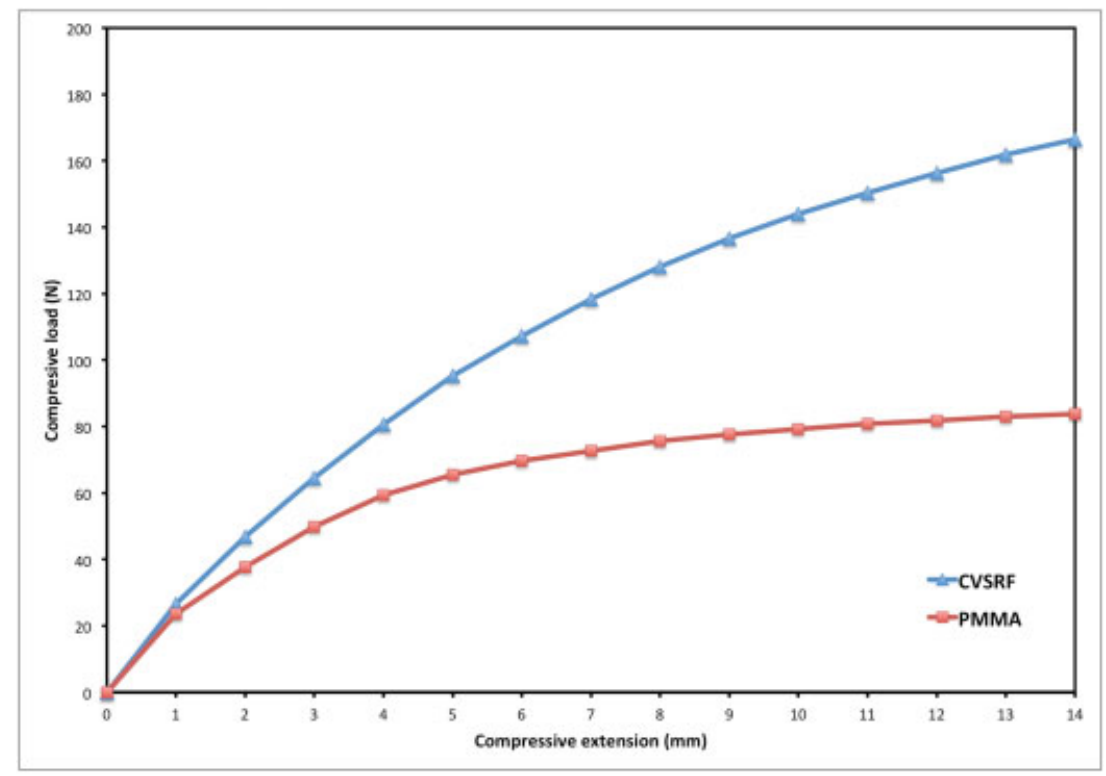

Fig. 3 The load-displacement curve for canine vertebral screw and rod fixation shows a higher rigidity and toughness than that for the screws combined with polymethylmethacrylate technique. CVSRF, canine vertebral screw and rod fixation; PMMA, polymethylmethacrylate. 


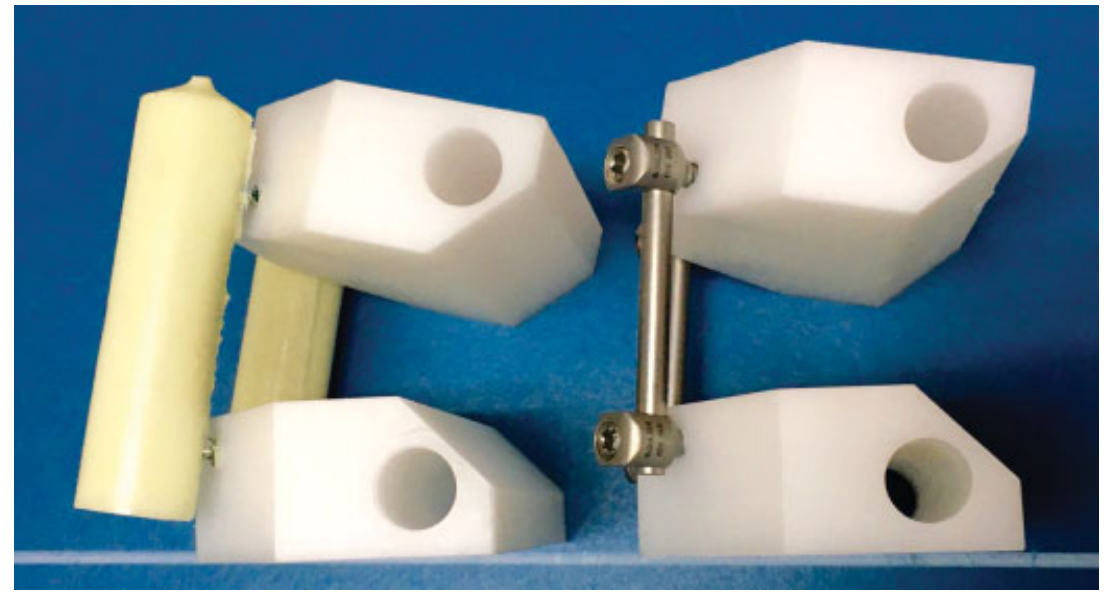

Fig. 4 After mechanical testing, the bending deformity was found at the insertion point in ultra-high-molecular-weight polyethylene blocks in both constructs. However, the rod and polymethylmethacrylate bar remained intact.

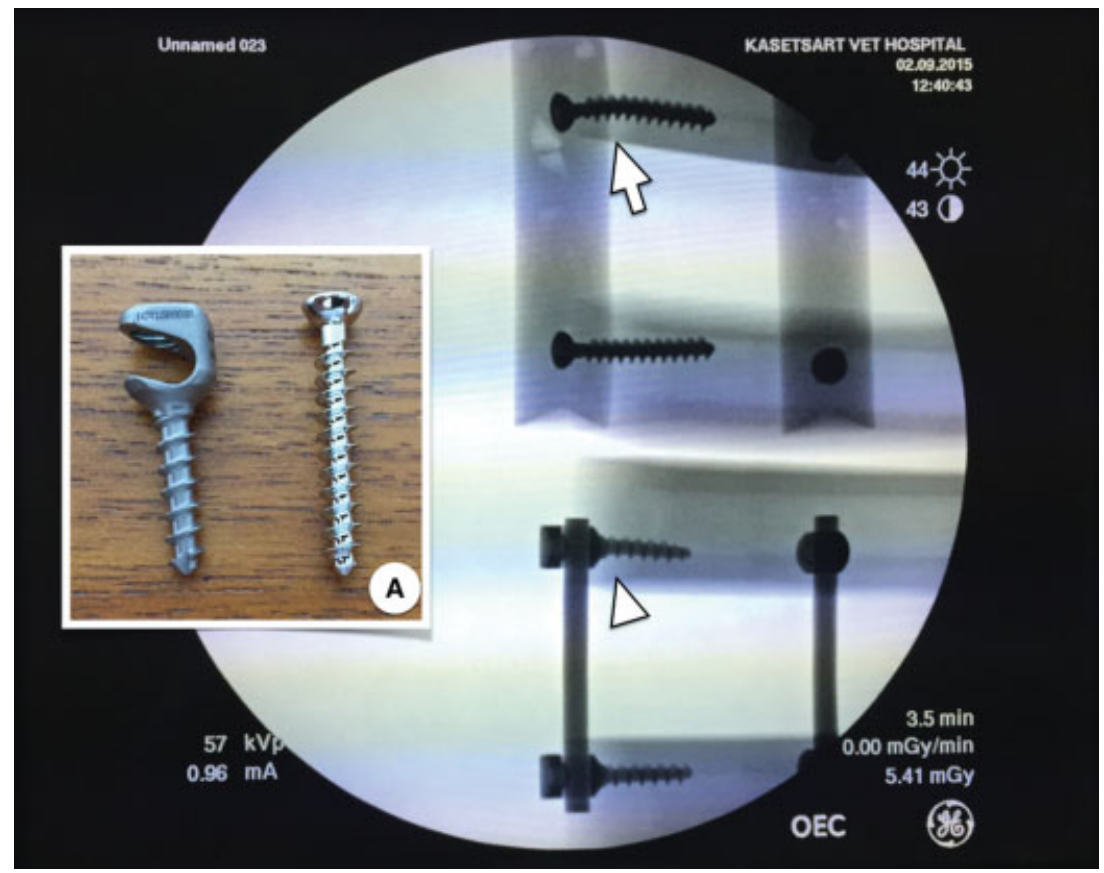

Fig. 5 Radiograph of the spinal assemblies after mechanical testing. The bending deformity at the insertion point of screw body in screws combined with polymethylmethacrylate construct (arrow) was visually larger (i.e., more bending) than in the canine vertebral screw and rod fixation prototype (arrowhead). (A) Comparison screw body of the 3.5-mm titanium vertebral screw in the canine vertebral screw and rod fixation prototype (left) and the $4.0-\mathrm{mm}$ stainless steel cancellous screw in the screws combined with polymethylmethacrylate technique (right).

\section{Discussion}

This study showed that a newly designed CVSRF has higher mechanical stiffness properties than the most commonly used construct in canine spinal fixation, namely the ScrewPMMA technique.

The monoaxial side-loaded screw head design was used in the CVSRF system instead of the top-loaded screw head design for humans due to the difference of screw head positioning on canine vertebrae. The top-loaded screw head was designed for humans because the pedicle screw trajectory is more accessible from the posterior side and perpendicular to the human vertebral column. In dogs, the anatomy of the lumbar spine allows an approach from the dorsolateral side to the pedicle and vertebral body and sideloading the rod on the screw head is more convenient. Insertion of the screws for the CVSRF system is different from insertion of pedicle screws. ${ }^{1}$ The side-loaded screw head was designed to fit easily on the dorsolateral surface of canine vertebrae and to facilitate insertion of the titanium rod on the screw head in the confined space of the spinal surgical field, thereby reducing trauma to the surrounding tissues. The 360-degree polyaxial screw head, which is popular in human spine surgery, also facilitates adaptation of the screw heads to the rods. The polyaxial screw head type is also top-loaded but is more expensive and has a reduced 
static compressive bending yield load compared with the monoaxial designs. ${ }^{7}$

The screws for the CSVRF system were designed as selftapping cancellous screw types with an outer cylindrical configuration and an inner conical configuration for maximum resistance to a pullout force. ${ }^{8}$ They can be used in various bone densities ${ }^{9}$ due to their design with the buttress thread and conical core. In this study, loosening of the selftapping 3.5 screws and the 4.0 cancellous screws from the UHMWPE blocks was not encountered. However, the variability of bone mineral density and size of vertebrae were eliminated by UHMWPE blocks. Thus, our test cannot predict how the system will perform in canine cadaveric models or in vivo, and for this further tests are necessary.

All the components of the CVSRF prototype were made of Ti6Al4V ELI. This material is accepted as an international standard for surgical implants in humans (ASTM F136-96). The Ti6Al4V ELI (Grade 23) is very similar to Ti6Al4V (Grade 5 ), but it provides improved ductility and better fracture toughness (ASTM F136-13). In addition, the histological biocompatibility of titanium in surrounding tissue is better than that of stainless steel and PMMA. ${ }^{10}$

In this study, the CVSRF screws with a diameter of $3.5 \mathrm{~mm}$ and length of $16 \mathrm{~mm}$ were chosen to represent the median screw size used in the thoracolumbar junction. Based on the report by Watine and colleagues, ${ }^{11}$ the length and width of the thoracolumbar implantation corridor were determined on computed tomography images from tenth thoracic to sixth lumbar vertebrae in 35 different adult dogs, with body weight between 4 and $75 \mathrm{~kg}$. The median length was $17.1 \mathrm{~mm}$ (range, $14-19 \mathrm{~mm}$ ) and the median width was $5.9 \mathrm{~mm}$ (range, $3-7 \mathrm{~mm}$ ). The screws were inserted approximately 50 to $80 \%$ into the vertebral body. ${ }^{3}$ In addition, the corticocancellous $3.5 \mathrm{~mm}$ screw was an appropriate sized implant for thoracolumbar vertebrae of dogs weighing 10 to $35 \mathrm{~kg}$.

According to ASTM F1717-04, static load types and dynamic tests should be performed for comparative evaluation of the new spinal implant assemblies. The main role of the screwrod system is to resist the compressive bending loads and to maintain stability of the spine in the sagittal plane in vivo. Although the anatomy of the canine and human spine are different, the biomechanical loading of the spine is remarkably similar between quadrupeds and bipeds. ${ }^{12}$ The most effective biomechanical force acting on the spine of the paralyzed canine patient is flexion. ${ }^{13}$ The greatest load placed on instrumentation postoperatively in paralyzed human patients is also flexion. ${ }^{14}$ Therefore, the CVSRF system was subjected primarily to static compressive bending testing. The fatigue test is considered as the dynamic test for new implants. This test was performed in a biomechanical study of a modified pedicle screw by Liu and colleagues, ${ }^{15}$ and the dynamic compressive tests showed that failure of the monoaxial screw bodies occurred at the insertion point into UHMWPE blocks.

The static test results indicated that the CVSRF system performed significantly better than the Screw-PMMA technique in terms of compressive bending yield load, compressive bending stiffness and compressive bending ultimate load. This may be explained by the specific CVSRF design contributing to its stiffness, namely the inner conical configuration of the vertebral screws, the side-loaded screw heads and the angular conformation of the inner screw resulting in a strong connection between rod and screws. This design makes the CVSRF system mechanically stronger than the Screw-PMMA technique.

Bending deformation occurred at the insertion point of the screw in both constructs after the mechanical testing while the rod and PMMA bar were still intact. Although the screw diameter $(4.0 \mathrm{~mm})$ in the PMMA construct was larger than that $(3.5 \mathrm{~mm})$ in the CVSRF construct, the bending defects at the neck of all CVSRF screws, as demonstrated by radiographic findings, were less than those of the screws in the PMMA constructs. The most probable explanation for this is the larger inner core screw design and the titanium component. ${ }^{16-18}$ The bending defects in the CVSRF screws may be resolved by increasing the screw diameter.

The pedicle screw and rod fixation system is reported as the surgical technique to solve the problems of the PMMA implants. ${ }^{3-6}$ However, the top-loaded head design is specifically designed for the human pedicle, and the available screw diameter that fits only in large breed dogs and its high cost limit the use of pedicle screw and rod fixation system in dogs. The CVSRF system was designed to solve this problem. The CVSRF system was tailored to the canine spine, and the screw diameter was adapted to the vertebral body to facilitate canine vertebral fixation. In addition, the mechanical properties of the CVSRF system were not different from the pedicle screw and rod fixation system in humans and were stronger than the Screw-PMMA technique.

The limitation of the present study was testing the device on UHMWPE blocks, which are different from canine vertebrae. Also, when applying the CVSRF system and the ScrewPMMA technique on the UHMWPE blocks, one can achieve the best match, which is difficult to meet during clinical surgical procedures. Therefore, our results cannot predict the biomechanical performance of these screw-rod systems in vivo. Evaluation of their use in a cadaveric setting and in clinical cases needs to demonstrate their efficacy. Also, a comparison of the failure loads to the expected loading in vivo would be useful to place the results into a meaningful clinical context. Ultimately, fatigue testing will be required to determine the endurance of both techniques.

In conclusion, the CVSRF is a new device that was designed and built to resolve the problem of current surgical technique for vertebral column fixation in dogs. All mechanical testing properties during bending showed that the CVSRF performed better than the Screw-PMMA technique.

Conflicts of Interest

The authors declared that they have no conflicts of interest in regard to this manuscript.

\section{Author Contributions}

P. Lewchalermwong and N. Suwanna contributed to the conception of the study, study design, and data acquisition. All authors contributed to data analysis and interpretation, drafting and revising as well as approval of the submitted manuscript. 


\section{References}

1 Jeffery ND. Vertebral fracture and luxation in small animals. Vet Clin North Am Small Anim Pract 2010;40(05):809-828

2 Gaines RW Jr. The use of pedicle-screw internal fixation for the operative treatment of spinal disorders. J Bone Joint Surg Am 2000;82-A(10):1458-1476

3 Meij BP, Suwankong N, Van der Veen AJ, Hazewinkel HA. Biomechanical flexion-extension forces in normal canine lumbosacral cadaver specimens before and after dorsal laminectomy-discectomy and pedicle screw-rod fixation. Vet Surg 2007;36(08): 742-751

4 Teunissen M, van der Veen AJ, Smit TH, Tryfonidou MA, Meij BP. Effect of a titanium cage as a stand-alone device on biomechanical stability in the lumbosacral spine of canine cadavers. Vet J 2017; 220:17-23

5 Smolders LA, Voorhout G, van de Ven R, et al. Pedicle screw-rod fixation of the canine lumbosacral junction. Vet Surg 2012;41 (06):720-732

6 Tellegen AR, Willems N, Tryfonidou MA, Meij BP. Pedicle screwrod fixation: a feasible treatment for dogs with severe degenerative lumbosacral stenosis. BMC Vet Res 2015;11:299

7 Stanford RE, Loefler AH, Stanford PM, Walsh WR. Multiaxial pedicle screw designs: static and dynamic mechanical testing. Spine 2004;29(04):367-375

8 Kim YY, Choi WS, Rhyu KW. Assessment of pedicle screw pullout strength based on various screw designs and bone densities-an ex vivo biomechanical study. Spine J 2012;12 (02):164-168
9 Krenn MH, Piotrowski WP, Penzkofer R, Augat P. Influence of thread design on pedicle screw fixation. Laboratory investigation. J Neurosurg Spine 2008;9(01):90-95

10 Yamaguchi K, Konishi H, Hara S, Motomura Y. Biocompatibility studies of titanium-based alloy pedicle screw and rod system: histological aspects. Spine J 2001;1(04):260-268

11 Watine S, Cabassu JP, Catheland S, Brochier L, Ivanoff S. Computed tomography study of implantation corridors in canine vertebrae. J Small Anim Pract 2006;47(11):651-657

$12 \mathrm{Smit} \mathrm{TH}$. The use of a quadruped as an in vivo model for the study of the spine - biomechanical considerations. Eur Spine J 2002;11 (02):137-144

13 Walker TM, Pierce WA, Welch RD. External fixation of the lumbar spine in a canine model. Vet Surg 2002;31(02):181-188

14 Jacobs RR, Nordwall A, Nachemson A. Reduction, stability, and strength provided by internal fixation systems for thoracolumbar spinal injuries. Clin Orthop Relat Res 1982;(171):300-308

15 Liu T, Zheng WJ, Li CQ, Liu GD, Zhou Y. Design and biomechanical study of a modified pedicle screw. Chin J Traumatol 2010;13(04): 222-228

16 Liu YK, Njus GO, Bahr PA, Geng P. Fatigue life improvement of nitrogen-ion-implanted pedicle screws. Spine 1990;15(04):311-317

17 Cho W, Cho SK, Wu C. The biomechanics of pedicle screw-based instrumentation. J Bone Joint Surg Br 2010;92(08):1061-1065

18 Hsu CC, Chao CK, Wang JL, Hou SM, Tsai YT, Lin J. Increase of pullout strength of spinal pedicle screws with conical core: biomechanical tests and finite element analyses. J Orthop Res 2005;23(04):788-794 